\title{
Some Children Do Need Psychotropic Medication
}

\section{Oswaldo Hector Chavez ${ }^{*}$}

National Board Certified Professional Counselor, Alabama, USA

Keywords: Medication; Children; Therapeutic interventions; Psychotropic

\section{Introduction}

I would like to discuss the current trend I am seeing as a clinician in regards to medication not being prescribed to children because the primary care doctor and/or psychiatrist refuses to medicate a child after therapeutic interventions have been tried for several months with little or no success, and have been short-lived due to the fact that the child has not stabilized from symptomatology of a classifiable DSM-5 diagnoses. This commentary does not suggest that the medical profession does not have the skills or the knowledge to address mental health issues as a whole. One of the main reasons for non-medicating is the actuality that it is a child and not an adult, which weighs heavily on the medical professional's psyche, as most of us do not think children require or need pharmaceutical assistance when they are at the beginning stages of their lives. I do understand that children do have developmental stages they must go through that occur over the child's life span. It is also well known and documented that children go through behavioral periods in life, more particularly during teenage years, although it is also experienced at younger ages. I think the problem really arises when medical professionals, specifically primary care doctors and certain psychiatrists, have not been given the appropriate training in medical school or during residency in working with children with mental health issues. From this de facto, they feel uncomfortable treating a child with depression, trauma, anxiety, etc. I have worked in many arenas and the unwillingness of practitioners refusing to medicate a child goes against the child's best interest, when it is medically indicated that the child meets the criteria for a medical diagnosis and can benefit from medication assistance. I have observed, in many instances, where children need medication to stabilize from depression in order for therapy / counseling to be effective, only to be prevented because the doctor feels that the child is too young for the medication. Recent research suggests that up to fifteen percent of children and adolescents have a mental disorder that is serious enough to cause some impairment, unfortunately, only one in five children receive services by appropriately trained mental health professionals. As a result, the child gets discharged from one counselor to the next, only to have the same issue arise of needing medication to stabilize. Parents end up having to take the child to their local ER to get temporary assistance, not long term help which they so badly need. On the other end of the spectrum, I have been at institutions in other states where I have been fortunate to have a child psychiatrist in-house who had the knowledge and the confidence in prescribing psychotropic medication to children combined with therapy, effectively stabilizing the child to receive services elsewhere.

Most of the research studies of psychotropic medication, intended to guide prescribers, have been done with adults with little or no studies for children. This gap of research knowledge creates a dilemma making psychiatrists and primary care doctors apprehensive in prescribing medication to a child. I would recommend that psychiatrist or primary care doctor to get additional training on child psychiatric medication to help ease the professional worries. I am not advocating for all children with mental health issues to be on medication, but I am saying that this is a noticeable trend I am seeing. Primary care doctors need to be aware that there is strong evidence of efficacy of cognitive behavioral therapy for issues such as anxiety in children; however, few doctors utilize SSRIs that may be effective for children who do not show improvement with behavioral therapy alone. Evidence also denotes that children and adolescents with depressive disorders respond well to a wide variety of psychosocial treatments. There are those who do not fit this category and Fluoxetine (Prozac) is the only medication that has been approved by the Food and Drug Administration for use in children, and it may increase the risk for suicidal behaviors in some children, again making it difficult for a psychiatrist or medical doctor to prescribe medication to a child because of the risk.

In general, medicating children can be a divisive topic--whether or not to turn to pharmaceuticals to cope with unexpected events and the emotions that arise from these events which cause parents to debate with school administrators, mental health professionals, family friends, politicians, caregivers and even themselves . There is no one-size-fits-all answer when dealing with the issue. Professionally, I feel medication can be an effective tool in the treatment of several psychiatric disorders of childhood and adolescence when all therapeutic attempts have failed. A doctor's recommendation to use medication often raises many concerns and questions in both the parents and the child--a prescribed medication may change how they interact, play, sleep, eat, and deal with everyday events. The psychiatrist or primary care doctor who recommends the medication should be experienced in treating psychiatric illnesses in children and adolescents. He or she should fully explain the reasons for medication use, what are the pros and cons, as well as possible risks, adverse effects, and other treatment alternatives.

\section{Autobiographical Summary}

Oswaldo H. Chavez acquired his Associates of Science in Pre Medical Studies from Dodge City Community College. He obtained his B.S. in Health and Human Performance and a Minor in Chemistry from Fort Hays State University. He went on to receive his Master's Degree from Texas A \& M University- Corpus Christi in Marriage and Family Counseling and Community Counseling. He received a full scholarship from the National Institute of Health for Biomedical Research to study Hispanic issues in Washington, DC. He completed his National Board Certification post-graduate certificate in counseling from the National

*Corresponding author: Oswaldo Hector Chavez, National Board Certified Professional Counselor, Licensed Marriage and Family Therapist Supervisor Licensed Professional Counselor Supervisor, and Licensed Drug and Alcohol Counselor, Po box 530386, Mountain Brook, AL 35223, USA, Tel: 361-7796223; E-mail: chavez461@gmail.com

Received September 11, 2015; Accepted December 16, 2015; Published December 22, 2015

Citation: Chavez OH (2016) Some Children Do Need Psychotropic Medication. J Psychiatry 18: 344 doi:10.4172/2378-5756.1000344

Copyright: (c) 2016 Chavez OH. This is an open-access article distributed under the terms of the Creative Commons Attribution License, which permits unrestricted use, distribution, and reproduction in any medium, provided the original author and source are credited 
Board for Certified Counselors in Greensboro, NC. He completed a six month selected deployment as a Clinical Research Counselor for the Army Combat Stress and Addiction Recovery Program Moncrieff Hospital, Fort Jackson, SC. He was selected due to his exceptional clinical and organizational skills. He has also served as a Clinical Counselor for the Navy Fleet and Family Support Center, Naval Station Ingleside, TX. Mr. Chavez is currently in private practice providing
Trauma Therapy to children who have been sexually, physically, and emotionally abused. Additionally, he provides crises therapy for the American Red Cross during weather disasters. He concurrently provides post deployment briefings and workshops on mental health issues to the Services of the Armed Forces via the American Red Cross. 